\title{
Temperature Effects on Dissociative Recombination in Neon
}

\author{
A. Urbanowicz, A. Bielsiki, D. Lisak, R. Ciuryło and R.S. Trawińshi* \\ Instytut Fizyki, Uniwersytet Mikołaja Kopernika, Grudziądzka 5/7, 87-100 Toruń, Poland
}

(Received July 26, 2010; in final form October 12, 2010)

\begin{abstract}
Using an interferometric method, detailed analysis of the line shapes of the self-broadened $748.8 \mathrm{~nm} \mathrm{Ne}$ line emitted from the glow discharge at low pressure of neon was performed in wide temperature range. Systematic departures from the ordinary Voigt profile were observed. We have shown that the dissociative recombination of molecular $\mathrm{Ne}_{2}^{+}$ions which gives rise to the non-thermalized neon atoms may be regarded as the main process responsible for the distortion of the profile of the investigated line.
\end{abstract}

PACS: 32.70.-n, 32.70.Jz

\section{Introduction}

Spectral line shape measurements of the self-broadened neon lines corresponding to the $\left(2 p^{5} 3 d-2 p^{5} 3 p\right)$ transitions emitted from the glow discharge at pressures about a few Torr have revealed systematic departures of the observed profiles from the Voigt profiles [1, 2]. These departures were satisfactorily explained [2] as caused by the dissociative recombination of molecular ions $\mathrm{Ne}_{2}^{+}$with electrons. Frommhold and Biondi [3] suggested, however, that also in the case of argon the process of dissociative recombination of molecular $\mathrm{Ar}_{2}^{+}$ions may influence the observed line shape.

In recent years a systematic study of the shapes of the $687.1 \mathrm{~nm}, 703.0 \mathrm{~nm}$ and $750.3 \mathrm{~nm}$ self-broadened argon lines as well as $540.0 \mathrm{~nm}$ and $748.8 \mathrm{~nm}$ neon lines emitted from low-current glow discharge sources has been performed in our laboratory using a pressure-scanned Fabry-Perot interferometer (FPI) [4-8]. For the $687.1 \mathrm{~nm}$ and $703.0 \mathrm{~nm}$ Ar lines and the $748.8 \mathrm{~nm}$ Ne line we have found that at pressures below 3 Torr the observed profiles differ markedly from the Voigt function. Following Stacey and Thompson [1] such departures were explained as due to effects associated with the mechanisms by which the emitting atoms are excited. We focused our attention on the dissociative recombination of molecular ions $\mathrm{X}_{2}^{+}(\mathrm{X}=\mathrm{Ar}, \mathrm{Ne})$ with electrons which can cause incomplete thermalization of the excited X-atoms [3, 9].

The dissociative recombination of $\mathrm{X}_{2}^{+}$ions with electrons is the reaction

$$
\begin{aligned}
& \mathrm{X}_{2}^{+}+\mathrm{e} \longrightarrow\left(\mathrm{X}_{2}^{+}\right)_{\text {unstable }} \longrightarrow \mathrm{X}^{*}+\mathrm{X} \\
& \quad+\text { kinetic energy },
\end{aligned}
$$

where the superscripts + and ${ }^{*}$ refer to ionized and excited states, respectively. In this reaction a molecular

\footnotetext{
* corresponding author; e-mail: rst@fizyka.umk.pl
}

rare-gas ion captures an electron to form an unstable excited molecule which then begins to dissociate producing two atoms, one in an excited state, moving apart with kinetic energy in excess of the kinetic energy corresponding to the gas temperature. In an earlier paper [2] this reaction was adapted as a basis of a model for the quantitative description of the departures of the observed profiles of the $753.5 \mathrm{~nm}$ and $754.4 \mathrm{~nm}$ neon lines from the Voigt profile. In paper [10] we applied this model to analyze the profiles of three lines of argon (687.1, 703.0 and $750.3 \mathrm{~nm}$ ) as well as the 540.0 and $748.8 \mathrm{~nm}$ lines of neon where for the first two argon lines and for the second neon line distortion of the profiles were observed.

The process of dissociative recombination in rare-gases which is schematically represented in Fig. 1 was thoroughly studied by Biondi and his coworkers $[3,9,11,12]$ who performed the high-resolution studies of line profiles to detect excess kinetic energy of dissociation fragments.

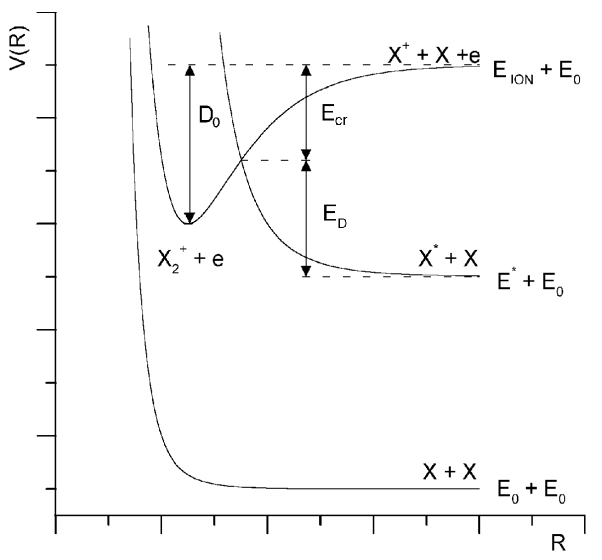

Fig. 1. The energy diagram of recombination process in rare gases. Notations explained in the text.

Connor and Biondi [9] and Frommhold and Biondi [3] have performed interferometric measurements of the profiles of many neon and argon lines emitted from a mi- 
crowave discharge and during the ensuing afterglow. They have found that in the afterglow the Doppler profiles are much broader than the thermal atom profiles of the lines observed in the discharge.

In our latest papers $[13,14]$ the shape of the self-broadened $748.8 \mathrm{~nm}$ neon line was analyzed in terms of speed-dependent asymmetric Voigt profile which takes into account correlations between collisional and Doppler broadening as well as line asymmetry. For the lower neon pressures we found, however, that the observed line profile is affected by an excitation process that could be the dissociative recombination of molecular ions $\mathrm{Ne}_{2}^{+}$with electrons. This result agreed very well with our earlier studies $[2,7,10]$ of the $748.8 \mathrm{~nm}$ Ne line, where we showed (see Fig. 6 in [10]) that the population fraction of non-thermalized atoms decreases with the neon pressure, and their role in the formation of the total line profile is negligible for neon pressures higher than 8 Torr. The line shape measurements presented in paper [13] were performed at room temperature of the gas discharge. In order to perform overall study of the influence of excitation process on the shape of the self-broadened $748.8 \mathrm{~nm}$ neon line in this paper we present the results of interferometric line shape measurements of this line done in wide temperature range.

\section{Spectral line shapes for non-thermalized excited atoms}

In the model described in [2] the dissociative recombination is assumed to be the main process responsible for the occurrence of non-thermalized excited atoms.

All dissociations which are due to dissociative-recombination process (Eq. (1)) which ends in a given excited atomic state originate in a single specific molecular state and therefore release a definite total kinetic energy $E_{\mathrm{D}}$ which is shared equally between two dissociating rare-gas atoms. This kinetic energy in excess of the kinetic energy of the thermal motion can be calculated from the formula $E_{\mathrm{D}}=E_{\mathrm{i}}-E^{*}-E_{\mathrm{cr}}$ where $E_{\mathrm{i}}$ is the ionization energy of the rare-gas atom, $E^{*}$ is the energy of a given excited state and $E_{\text {cr }}$ is the energy for which the crossing of potential curves of the $\mathrm{X}^{*}-\mathrm{X}$ and $\mathrm{X}^{+}-\mathrm{X}$ systems occurs (see Fig. 1).

The speed $v_{\mathrm{D}}$ of the excited $\mathrm{Rg}^{*}$ atom relative to the center of mass of the diatomic ion $\mathrm{Rg}_{2}^{+}$is given by the formula $v_{\mathrm{D}}=\left(E_{\mathrm{D}} / m\right)^{1 / 2}$, with $m$ being the mass of a single rare-gas atom. It should be noted that for a given $E_{\mathrm{D}}$ energy the absolute value of $v_{\mathrm{D}}$ is fixed but the direction taken by the excited rare-gas atom is randomly oriented in the reference frame. It leads to the rectangular form of the excess Doppler profile caused by excess kinetic energy due to the dissociative recombination process [2]. To obtain the resultant Doppler profile of a spectral line emitted by non-thermalized excited atoms this rectangular profile is convoluted with the Doppler profile corresponding to the thermal motion of the center of mass of the diatomic ion $\mathrm{Rg}_{2}^{+}$.
Using the results from [6] one can obtain the FPI response function $O_{\mathrm{nth}}(\widetilde{\nu})$ describing the shape of the registered spectral line emitted by non-thermalized excited atoms, i.e. those atoms that are produced due to dissociative recombination processes, which can be written in the following way:

$$
\begin{aligned}
& O_{\text {nth }}(\widetilde{\nu})=\frac{1}{\Omega}\left[\frac{1}{2}+\sum_{n=1}^{\infty} R^{n} \mathrm{e}^{-n L} \mathrm{e}^{-\frac{n^{2} D^{2}}{8}} \mathrm{e}^{-\frac{n^{2} G^{2}}{4}}\right. \\
& \left.\quad \times \operatorname{sinc}(n B) \cos \left(\frac{2 n \pi}{\Omega}\left(\widetilde{\nu}-\widetilde{\nu}_{0}-\Delta\right)\right)\right],
\end{aligned}
$$

where $\Omega$ is the free spectral range of FPI, $R$ is the reflection coefficient of the FPI mirrors and $\widetilde{\nu}_{0}$ is the unperturbed wave number. In the above equation $L=\pi \gamma_{\mathrm{L}} / \Omega$ is the reduced Lorentzian width, $G=\pi \gamma_{\mathrm{G}} /(\Omega \sqrt{\ln 2})$ is the reduced width of the Gaussian component of the instrumental function of the real FPI, $D=\pi \gamma_{\mathrm{D}} /(\Omega \sqrt{\ln 2})$ is the reduced Doppler width and $\operatorname{sinc}(y)=\sin (y) / y$. $B=\pi \gamma_{\mathrm{DIS}} / \Omega$ is the reduced dissociative width and $\gamma_{\mathrm{DIS}}=2 \widetilde{\nu}_{0} \sqrt{\frac{E_{\mathrm{D}}}{m c^{2}}}$.

The total profile $O_{\mathrm{VDP}}(\widetilde{\nu})$, which is the response of the Fabry-Perot interferometer to the Voigt dissociative profile (VDP), is constructed from the thermalized and non-thermalized profiles and can be written as [2]:

$$
\begin{gathered}
O_{\mathrm{VDP}}(\widetilde{\nu})=\eta_{\mathrm{th}} O_{\mathrm{th}}(\widetilde{\nu})+\eta_{\mathrm{nth}} O_{\mathrm{nth}}(\widetilde{\nu}) \\
=\frac{1}{1+\eta}\left[O_{\mathrm{th}}(\widetilde{\nu})+\eta O_{\mathrm{nth}}(\widetilde{\nu})\right]
\end{gathered}
$$

with $\eta=N_{\text {nth }} / N_{\text {th }}$.

Here $N_{\text {th }}$ and $N_{\text {nth }}$ denote the number densities of the thermalized and non-thermalized excited atoms, respectively. The population fraction in each case is $\eta_{\text {nth }}=$ $N_{\text {nth }} / N_{0}$ and $\eta_{\text {th }}=N_{\text {th }} / N_{0}$, where $N_{0}=N_{\text {nth }}+N_{\text {th }}$.

It is seen that the total profile consists of two components: a thermal component $O_{\text {th }}(\widetilde{\nu})$ expressed in terms analogous to the Ballik formula [15], being the response of the ideal FPI to the ordinary Voigt profile $\left(O_{\mathrm{th}}(\widetilde{\nu})=\right.$ $\left.O_{\mathrm{VP}}(\widetilde{\nu})\right)$ where

$$
\begin{aligned}
& O_{\mathrm{VP}}(\widetilde{\nu})=\frac{1}{\Omega}\left[\frac{1}{2}+\sum_{n=1}^{\infty} R^{n} \mathrm{e}^{-n L} \mathrm{e}^{-\frac{n^{2} D^{2}}{4}} \mathrm{e}^{-\frac{n^{2} G^{2}}{4}}\right. \\
& \left.\quad \times \cos \left(\frac{2 n \pi}{\Omega}\left(\widetilde{\nu}-\widetilde{\nu}_{0}-\Delta\right)\right)\right],
\end{aligned}
$$

and a broader, non-thermal component $O_{\text {nth }}(\widetilde{\nu})$ of the form given by formula Eq. (2).

\section{Results and discussion}

The experimental details concerning apparatus setup and measurement procedure have been described in detail in our earlier paper [13]. The experimental intensity distributions of the self-broadened $748.8 \mathrm{~nm}$ Ne line were fitted directly to the responses of the Fabry-Perot interferometer $O(\widetilde{\nu})$ for different theoretical profiles. In 

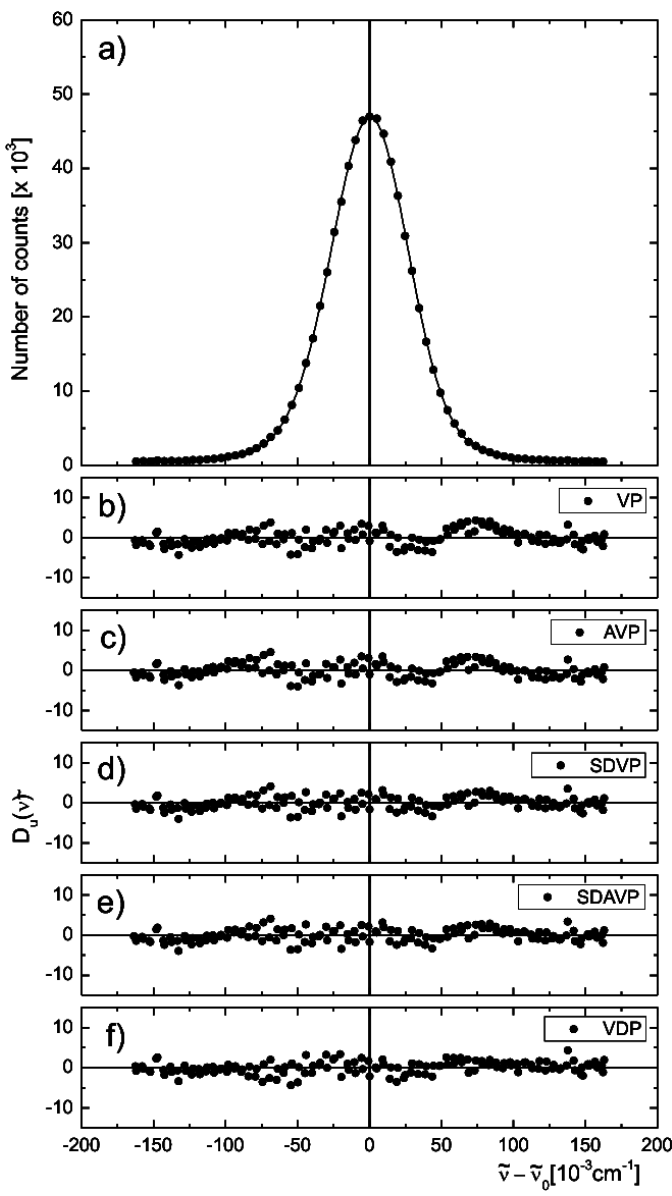

Fig. 2. The shape of the self-broadened $748.8 \mathrm{~nm} \mathrm{Ne}$ line perturbed by neon at temperature $654 \mathrm{~K}$ and pressure of 8.2 Torr: (a) experimental points together with the best-fit VDP profile (full curve), (b)-(f) weighted differences $D_{\mathrm{u}}(\widetilde{\nu})$ between experimental and fitted VP, AVP, SDVP, SDAVP and VDP profiles, respectively.

line shape analysis we use the following theoretical profiles: ordinary Voigt profile (VP), speed-dependent Voigt profile (SDVP), asymmetric Voigt profile (AVP), speed-dependent asymmetric Voigt profile (SDAVP). In line shape analysis presented in this paper we used all the above theoretical profiles together with VDP which takes into account effects due to excitation process.

As an example of the measured profiles Fig. 2 shows the shape of the self-broadened $748.8 \mathrm{~nm}$ Ne perturbed by neon at temperature $654 \mathrm{~K}$ and pressure of 8.2 Torr. Similar to our previous studies for noble gas atoms we have used a Marquardt [16] least-squares algorithm for nonlinear parameters to perform the best-fit procedures.

As in paper [13] we started the analysis with the ordinary Voigt profile $O_{\mathrm{VP}}(\widetilde{\nu})$ in which neither collision-time asymmetry nor correlation effects were taken into account, since in this case the fit procedure reveals all the departures. In order to examine the quality of the fits we used the weighted differences of the intensities, $D_{\mathrm{u}}(\widetilde{\nu})=\left[I_{\exp }(\widetilde{\nu})-I_{\text {fit }}(\widetilde{\nu})\right] / u(\widetilde{\nu})$, between experimental

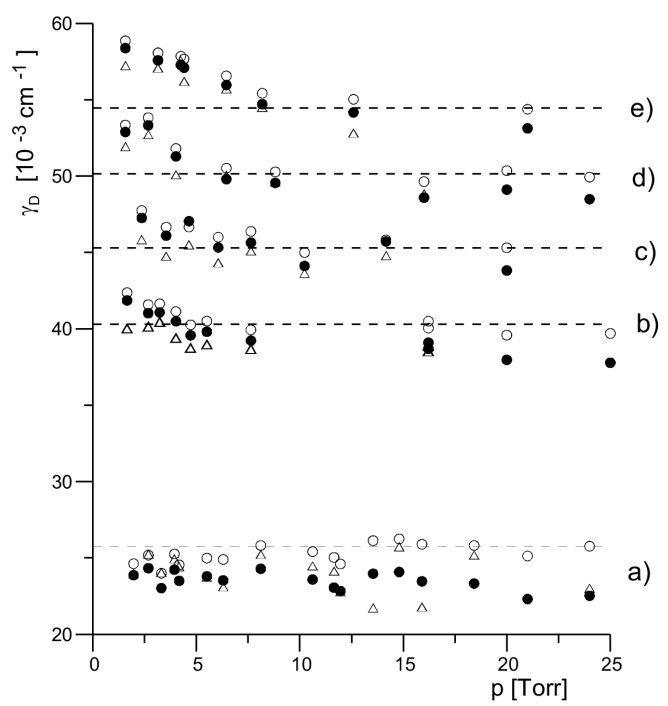

Fig. 3. Plots of the Doppler width $\gamma_{\mathrm{D}}$ of the $748.8 \mathrm{~nm}$ Ne line perturbed by neon determined from the best fit of the AVP profile (full circles), SDAVP profile (open circles), VDP (triangles) to the experimental data for investigated temperatures (cases (a-e) see Table), against the pressure $p$ of Ne. Dashed line - mean Doppler width. For clarity the error bars are not plotted in the figure. The standard uncertainty values are of the magnitude of plotted symbols.

(measured) $I_{\exp }(\widetilde{\nu})$ and fitted (theoretical) $I_{\text {fit }}(\widetilde{\nu})$ profiles, where $u(\widetilde{\nu})$ is the uncertainty of the measured signal.

In Fig. 2b we can see systematic departures from zero in the line core as well as on line wings, which are typical for lower pressures (about a few Torr) of perturbing gas.

In the next steps we have fitted asymmetric Voigt profiles $O_{\text {AVP }}(\widetilde{\nu})$, speed-dependent Voigt profiles $O_{\mathrm{SDVP}}(\widetilde{\nu})$ and speed-dependent asymmetric Voigt profiles $O_{\mathrm{SDAVP}}(\widetilde{\nu})$. We can see a gradual improvement of the fit quality but there are still systematic departures in the differences plots - Figs. 2c-e.

Finally, we have fitted the $O_{\mathrm{VDP}}(\widetilde{\nu})$ profile $(\mathrm{Eq} .(3))$ taking into account both the uncorrelated collisional and Doppler broadening and the influence of radiation emitted from non-thermalized neon atoms. As can be seen from Fig. $2 \mathrm{f}$ in this case the values of the differences are spread uniformly about zero which confirms the goodness of the fit. It is thus shown that in the case of lower perturbing gas pressures the observed line profile is affected by an excitation process that could be the dissociative recombination of molecular ions $\mathrm{Ne}_{2}^{+}$with electrons. This result agrees very well with our earlier studies $[2,7$, $10,13,14]$ of the $748.8 \mathrm{~nm}$ Ne line, where we showed (see Fig. 6 in [10]) that the population fraction of non-thermalized atoms decreases with the neon pressure, and their role in the formation of the total line profile $(3)$ is negligible for neon pressures higher than 8 Torr.

A numerical fit of experimental profiles to $O_{\mathrm{VP}}(\widetilde{\nu})$ as well as to $O_{\operatorname{SDVP}}(\widetilde{\nu})$ allowed three parameters to vary: the Lorentzian width $\gamma_{\mathrm{L}}$, the pressure shift $\Delta$ and the 
Gaussian width $\gamma_{\mathrm{D}}$. For $O_{\mathrm{AVP}}(\widetilde{\nu})$ and $O_{\mathrm{SDAVP}}(\widetilde{\nu})$ we also fitted the collision-time asymmetry parameter $\chi$.

In Fig. 3 the Doppler widths $\gamma_{D}$ of the self-broadened $748.8 \mathrm{~nm}$ Ne line are plotted against Ne pressure in the pressure range up to 25 Torr. In this figure we plotted the values of the Doppler widths $\gamma_{\mathrm{D}}$ determined by fitting the $O_{\mathrm{AVP}}(\widetilde{\nu})$ (full circles) and $O_{\mathrm{SDAVP}}(\widetilde{\nu})$ (open circles) formulae to the experimental profile. The mean values of $\overline{\gamma_{\mathrm{D}}}$ are marked as dashed lines and are listed together with appropriate mean Doppler temperatures $\overline{T_{\mathrm{D}}}$ in Table.

\section{TABLE}

Experimental values of the mean dissociative width $\overline{\gamma_{\text {DIS }}}$ (in units $10^{-3} \mathrm{~cm}^{-1}$ ), kinetic energy in excess of the kinetic energy of the thermal motion $E_{\mathrm{D}}$, and energy of crossing of potential curves $E_{\mathrm{cr}}$ (in $\mathrm{eV}$ ) determined for investigated temperatures together with mean Doppler widths $\overline{\gamma_{\mathrm{D}}}$ (in units $10^{-3} \mathrm{~cm}^{-1}$ ) and mean Doppler temperatures $\overline{T_{\mathrm{D}}}$ (in $\mathrm{K}$ ). $D_{0}$ is the dissociation energy of the $\mathrm{Ne}_{2}^{+}$molecular ion (in $\mathrm{eV}$ ). Data in last row are taken from our earlier papers [7, 10]. For experimental data the values of standard uncertainties are given.

\begin{tabular}{c|c|c|c|c|c|c}
\hline \hline Case & $\overline{\gamma_{\mathrm{D}}}$ & $\overline{T_{\mathrm{D}}}$ & $\overline{\gamma_{\mathrm{DIS}}}$ & $E_{\mathrm{D}}$ & $E_{\text {cr }}$ & $D_{0}$ \\
\hline $\mathrm{a}$ & $25.7 \pm 1.1$ & $146 \pm 14$ & $80 \pm 32$ & $0.17 \pm 0.35$ & $1.36 \pm 0.35$ & $1.291 \pm 0.010$ \\
$\mathrm{~b}$ & $40.3 \pm 0.6$ & $358 \pm 11$ & $134 \pm 10$ & $0.48 \pm 0.07$ & $1.05 \pm 0.07$ & $1.291 \pm 0.010$ \\
$\mathrm{c}$ & $45.3 \pm 0.4$ & $454 \pm 8$ & $140 \pm 6$ & $0.52 \pm 0.04$ & $1.01 \pm 0.04$ & $1.291 \pm 0.010$ \\
$\mathrm{~d}$ & $50.2 \pm 0.7$ & $553 \pm 15$ & $143 \pm 5$ & $0.55 \pm 0.03$ & $0.98 \pm 0.03$ & $1.291 \pm 0.010$ \\
$\mathrm{e}$ & $54.5 \pm 0.6$ & $654 \pm 14$ & $145 \pm 12$ & $0.56 \pm 0.07$ & $0.97 \pm 0.07$ & $1.291 \pm 0.010$ \\
\hline$[7,10]$ & $38.3 \pm 1.3$ & $320 \pm 20$ & $124 \pm 17$ & $0.41 \pm 0.12$ & $1.12 \pm 0.12$ & $1.291 \pm 0.010$
\end{tabular}

The values of $\gamma_{\mathrm{D}}$ marked by triangles in Fig. 3 were determined from fitting of the $O_{\mathrm{VDP}}(\widetilde{\nu})$ profile (Eq. (3)) to the experimental line profiles in the range of neon pressures up to 7 Torr. It is seen that the inclusion to the fitted line profile $O_{\mathrm{VDP}}(\widetilde{\nu})$ of the contributions due to non-thermalized neon atoms leads here to a systematic decrease of the determined Doppler widths $\gamma_{\mathrm{D}}$, which in most cases are now closer to the mean $\overline{\gamma_{\mathrm{D}}}$ value.

In Fig. 4 open circles show the plots of the Lorentzian width $\gamma_{\mathrm{L}}$ determined from the best fit of the $O_{\mathrm{SDAVP}}(\widetilde{\nu})$ profile to our experimental profiles against the density number $N$ of neon for different discharge temperatures. As triangles we have plotted here the values of the Lorentzian width $\gamma_{\mathrm{L}}$ determined from the best fit of the $O_{\mathrm{VDP}}(\widetilde{\nu})$ profile to our experimental data. As shown in Fig. 4 the values of $\gamma_{\mathrm{L}}$ are in both cases linearly dependent on the density, but the values of residual Lorentzian width in the limit of zero density are always smaller and closer to zero for the $O_{\mathrm{VDP}}(\widetilde{\nu})$ profile. It is in agreement with our earlier observations for $753.5 \mathrm{~nm}$ and $754.4 \mathrm{~nm}$ neon lines [2] where the inclusion of the dissociative-recombination effects explained the anomalous behavior of their Lorentzian width. We do not present the determined values of residual Lorentzian widths of $748.8 \mathrm{~nm}$ line as their accuracy depends strongly on the accuracy of determination of the reflection coefficient of the Fabry-Perot plates. It is seen from Eqs. (2) and (4) that the change of the reflection coefficient (for example due to ageing process) equal to $\Delta R$ leads to the change of Lorentzian width $\Delta \gamma_{\mathrm{L}}=-(\Omega / \pi) \ln (1+\Delta R / R)$.

Figure 5 shows the values of parameters $\eta=N_{\text {nth }} / N_{\text {th }}$ determined from the best-fit of $O_{\mathrm{VDP}}(\widetilde{\nu})$ profile to the

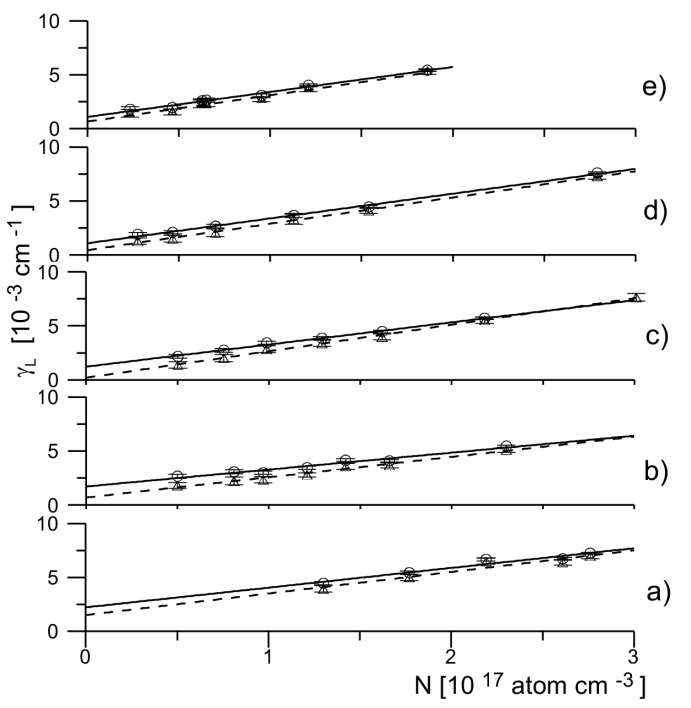

Fig. 4. Plots of the Lorentzian width $\gamma_{\mathrm{L}}$ of the $748.8 \mathrm{~nm}$ Ne line determined from the best fit SDAVP (open circles) and VDP (triangles) profiles for investigated temperatures against the neon density $N$. Error bars indicate the value of the standard uncertainty. Cases $(\mathrm{a}-\mathrm{e})$ see Table.

shapes of self-broadened $748.8 \mathrm{~nm}$ neon line measured at different discharge temperatures. The $\eta$-values decrease with increasing neon pressure which indicates that at higher pressures the broadening effects due to dissociative-recombination become negligible and all excited atoms may be treated as thermalized. 


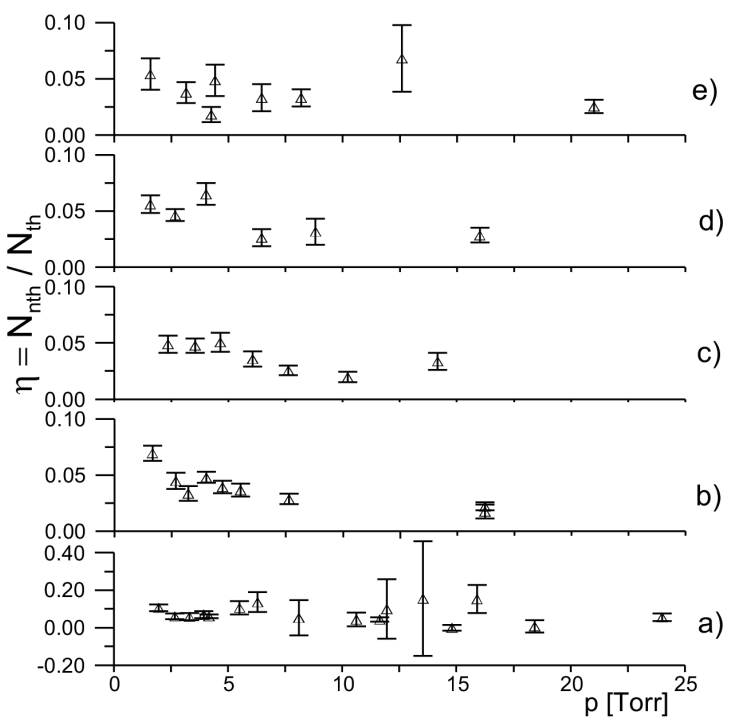

Fig. 5. Plots of the $\eta=N_{\text {nth }} / N_{\text {th }}$ parameters determined from the best-fit of $O_{\mathrm{VDP}}(\widetilde{\nu})$ profile to the shapes self-broadened $748.8 \mathrm{~nm}$ neon line measured at different discharge temperatures against the neon pressure $p$. Error bars indicate the value of the standard uncertainty. Cases $(\mathrm{a}-\mathrm{e})$ see Table.

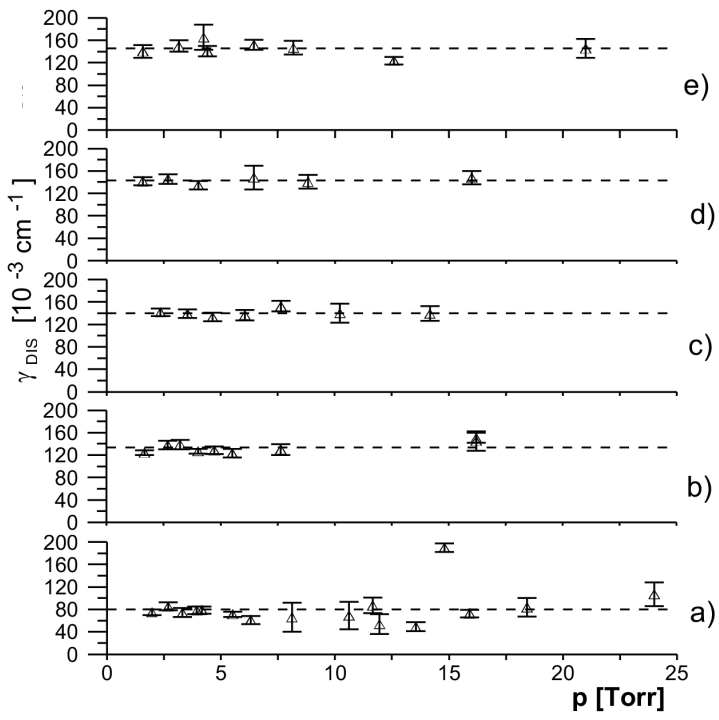

Fig. 6. Plots of the dissociative width $\gamma_{\text {DIS }}$ determined from the best-fit of $O_{\mathrm{VDP}}(\widetilde{\nu})$ profile to the shapes self-broadened $748.8 \mathrm{~nm}$ neon line measured at different discharge temperatures against the neon pressure $p$. Dashed line - mean dissociative width $\overline{\gamma_{\text {DIS }}}$. Error bars indicate the value of the standard uncertainty. Cases $(\mathrm{a}-\mathrm{e})$ see Table.

Figure 6 shows that for all investigated neon discharge temperatures the dissociative width $\gamma_{\text {DIS }}$ does not depend on the perturbing gas pressure which agrees with theoretical predictions $[2,3]$. The values of mean dissociative width $\overline{\gamma_{\text {DIS }}}$, listed in Table and plotted in Fig. 7 , increase with the neon discharge temperature. In Fig. 7 we also plotted one result obtained for self-broadened $748.8 \mathrm{~nm}$

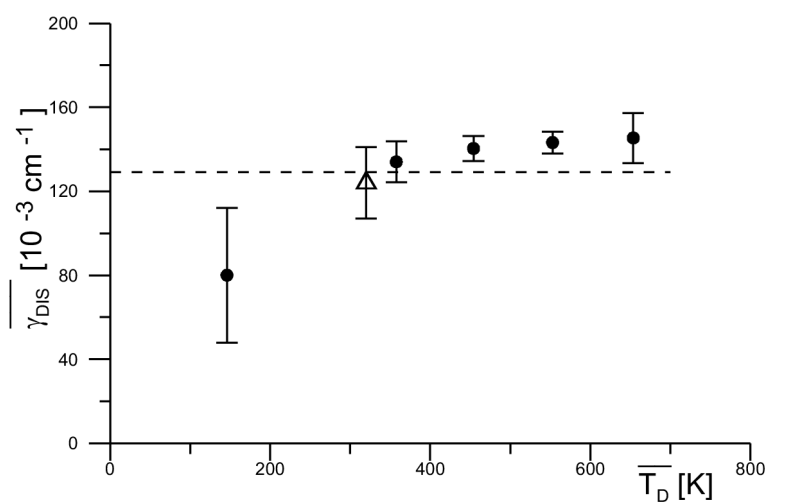

Fig. 7. Plots of the mean dissociative width $\overline{\gamma_{\text {DIS }}}$ against the mean Doppler temperatures $\overline{T_{\mathrm{D}}}$ (experimental points: full circles - this paper, triangle - $[7,10])$. Error bars indicate the value of the standard uncertainty.

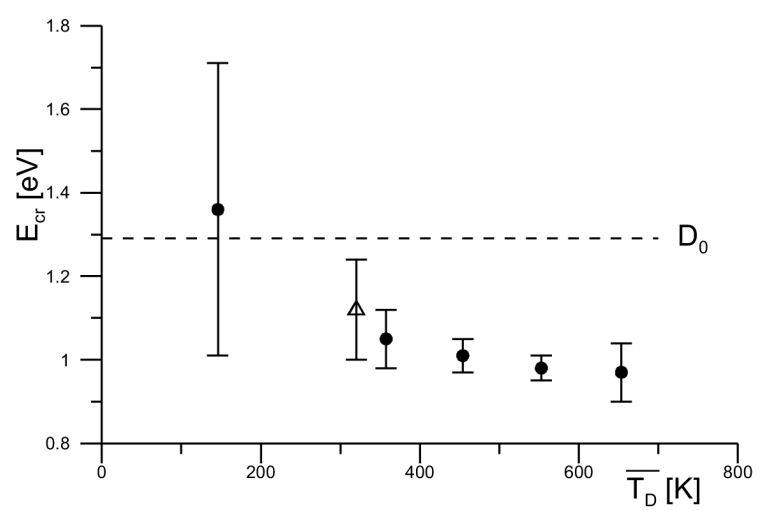

Fig. 8. Plots of the energy of crossing of potential curves $E_{\text {cr }}$ against the mean Doppler temperatures $\overline{T_{\mathrm{D}}}$ (experimental points: full circles - this paper, triangle - $[7,10]) . D_{0}$ is the dissociation energy of the molecular ion $\mathrm{Ne}_{2}^{+}$in its ground electronic state. Error bars indicate the value of the standard uncertainty.

neon line in the course of the earlier studies [7, 10] using different discharge lamp of an earlier construction [17]. Using the best fit values of mean dissociative width $\overline{\gamma_{\text {DIS }}}$ determined in the present work, for all investigated temperatures, we have found the appropriate values $E_{\mathrm{D}}$ of the kinetic energy of neon atoms in excess of the kinetic energy of the thermal motion and listed them in Table. From these values we finally calculated the values of $E_{\text {cr }}$ for which the crossing of potential curves of the $\mathrm{Ne}^{*}-\mathrm{Ne}$ and $\mathrm{Ne}^{+}-\mathrm{Ne}$ occurs (see Fig. 1) and listed them in Table and plotted in Fig. 8.

It should be noted that the values of energy $E_{\text {cr }}$ monotonically increase with the decrease of the discharge temperature, but on the other hand the value of its uncertainty for lowest temperature is so high that in the range of even one standard uncertainty we can treat the average $E_{\text {cr }}$ value as independent of the temperature. Moreover it fulfils the condition $E_{\text {cr }} \leq D_{0}$, where 
$D_{0}=(1.291 \pm 0.010) \mathrm{eV}[18]$ is the dissociation energy of the molecular ion $\mathrm{Ne}_{2}^{+}$in its ground electronic state.

\section{Concluding remarks}

In the present study we have shown that for self-broadened $748.8 \mathrm{~nm}$ neon line emitted from the glow discharge, measured in wide temperature range, for the neon pressures below 25 Torr systematic departures of the registered line shape from the Voigt profile occur. We have also shown that these departures may be interpreted assuming that the dissociative recombination of $\mathrm{Ne}_{2}^{+}$ions which gives rise to non-thermalized neon atoms is the main process responsible for the distortion of the line profiles.

It should be noted, however, that similar distortion of the line profile may be caused by any other process (e.g. [19]) additive to the thermal motion of atoms leading to the rectangular distribution of the excess speed.

\section{References}

[1] D.N. Stacey, R.C. Thompson, J. Phys. B 16, 537 (1983).

[2] R. Ciuryło, A. Bielski, J. Domysławska, J. Szudy, R.S. Trawiński, J. Phys. B 27, 4181 (1994).

[3] L. Frommhold, M.A. Biondi, Phys. Rev. 185, 244 (1969).

[4] J. Wawrzyński, J. Wolnikowski, Phys. Scr. 33, 113 (1986).
[5] A. Bielski, J. Wawrzyński, J. Wolnikowski, Acta Phys. Pol. A 67, 621 (1985).

[6] R. Ciuryło, Ph.D. Thesis, Nicolas Copernicus University, Toruń 1998.

[7] R. Ciuryło, A. Bielski, S. Brym, J. Domysławska, D. Lisak, J. Szudy, R.S. Trawiński, Acta Phys. Pol. A 96, 359 (1999).

[8] A. Bielski, S. Brym, R. Ciuryło, J. Szudy, Eur. Phys. J. D 8, 177 (2000).

[9] T.R. Connor, M.A. Biondi, Phys. Rev. 140, A778 (1965).

[10] A. Bielski, R. Ciuryło, D. Lisak, R.S. Trawiński, Acta Phys. Pol. A 97, 275 (2000).

[11] A.M. Biondi, Phys. Rev. 129, 1181 (1963).

[12] W.A. Rogers, M.A. Biondi, Phys. Rev. 134 A, 1215 (1964).

[13] A. Urbanowicz, A. Bielski, D. Lisak, R. Ciuryło, R.S. Trawiński, Eur. Phys. J. D 56, 17 (2010).

[14] A. Urbanowicz, A. Bielski, D. Lisak, R. Ciuryło, R.S. Trawiński, Eur. Phys. J. D 61, 1 (2011).

[15] E.A. Ballik, Appl. Opt. 5, 170 (1966).

[16] D.W. Marquardt, J. Soc. Industr. Appl. Math. 11, 431 (1963).

[17] A. Bielski, J. Wolnikowski, Acta Phys. Pol. A 54, 601 (1978).

[18] R.I. Hall, Y. Lu, Y. Morioka, T. Matsui, T. Tanaka, H. Yoshii, T. Hayaishi, K. Ito, J. Phys. B 28, 2435 (1995).

[19] J.F. Su, J.L. Nicol, J. Phys. B 29, 4093 (1996). 